\title{
Two Very Accurate and Efficient Methods for Solving Time-Dependent Problems
}

\author{
Mohamed El-Gamel, Waleed Adel, M. S. El-Azab
}

Department of Mathematical Sciences, Faculty of Engineering, Mansoura University, Mansoura, Egypt

Email: gamel_eg@yahoo.com

How to cite this paper: El-Gamel, M., Adel, W. and El-Azab, M.S. (2018) Two Very Accurate and Efficient Methods for Solving Time-Dependent Problems. Applied Mathematics, 9, 1270-1280.

https://doi.org/10.4236/am.2018.911083

Received: October 2, 2018

Accepted: November 18, 2018

Published: November 21, 2018

Copyright (c) 2018 by authors and Scientific Research Publishing Inc.

This work is licensed under the Creative Commons Attribution International License $(\mathrm{CC}$ BY 4.0).

http://creativecommons.org/licenses/by/4.0/

\section{Open Access}

\begin{abstract}
In this paper, collocation method based on Bernoulli and Galerkin method based on wavelet are proposed for solving nonhomogeneous heat and wave equations. The two methods have the linear systems solved by suitable solvers. Several examples are given to examine the performance of these methods and a comparison is made.
\end{abstract}

\section{Keywords}

Wavelet, Galerkin, Daubechies, Bernoulli, Collocation

\section{Introduction}

Recently, there has been a great deal of interest in "global" methods (Galerkin and collocation methods) for the numerical solution of twopoint boundary value problems. By this, we mean methods which find a solution in the form

$$
u_{N}(x)=\sum_{k=1}^{N} a_{k} \psi_{k}(x)
$$

where $\left\{\psi_{k}(x)\right\}_{k=1}^{N}$ is a basis for an $N$-dimensional subspace of functions, $S$. The functions $\psi_{k}(x), k=1,2, \cdots, N$, are called test functions and the space $S$ is called the test space.

To simplify the computations, the basis test functions $\left\{\psi_{k}(x)\right\}_{k=1}^{N}$ are taken to be orthogonal and in many cases they are polynomials, splines, sinc or wavelet. In essence, the Galerkin method is a discretization scheme in which the expansion coefficients $\left\{a_{k}\right\}_{k=1}^{N}$ are obtained by solving a set of $N$ algebraic equations.

For example, consider the problem $L u=f$, where $L$ is a self-adjoint operator and $f$ is a known function. The Galerkin method yields the system of equations

$$
\sum_{k=1}^{N} a_{k}\left\langle L \psi_{k}(x), \psi_{j}(x)\right\rangle=\left\langle f(x), \psi_{j}(x)\right\rangle, \quad j=1, \cdots N,
$$


which is an algebraic system of equations that can be solved for the unknown coefficients $a_{k}$.

In the collocation method no quadrature sums are required. The unknown coefficients $a_{k}$ in (1) are determined by the linear system

$$
L u_{N}\left(x_{j}\right)=f\left(x_{j}\right), \quad 0<x_{j}<1, \quad j=1,2, \ldots, m
$$

The aim of this paper is to develop the wavelet and Bernoulli bases for solving PDE of the form

$$
\frac{\partial^{\alpha} u}{\partial t^{\alpha}}=\frac{\partial^{2} u}{\partial x^{2}}+\eta(x, t), \quad 0 \leq x \leq 1, \quad t>0,
$$

subject to the boundary conditions

$$
u(0, t)=h_{1}(t), \quad u(1, t)=h_{2}(t),
$$

and the initial conditions

$$
\begin{aligned}
u(x, 0) & =f(x) \\
(\alpha-1) u_{t}(x, 0) & =(\alpha-1) g(x)
\end{aligned}
$$

where $\alpha \in\{1,2\}$.

In this paper, there are two different approaches for solving twopoint boundary value problems. Galerkin is based on wavelet and collocation is based on using Bernoulli operational matrix to reduce the problem into solving a system of linear algebraic equations. Recently, there has been a lot of research papers dealing with waveletGalerkin. Those papers include solution of partial differential equations [1], two-point boundary value problems [2], integro-differential equations [3], second-kind integral equations [5], Fredholm integral equations numerically [4,6], nonhomogeneous time-dependent problems [7], singularly Perturbed convection-dominated diffusion equation [8], telegraph equations [9], eigenvalue problem of a compact integral operator [10], fourth-order multi-dimensional elliptic partial differential equations [11], fourth order linear and nonlinear differential equations [12], stochastic fractional differential equations [13], Schordinger equations with general nonlinearity [14], generalized wavelet-Galerkin method [15]. El-Gamel et al. [16], have compared the wavelet-Galerkin and sinc-Galerkin techniques in solving nonhomogeneous heat equations. Moreover, El-Gamel has compared the wavelet-Galerkin and Adomian decomposition methods of boundary-value problems [17].

Bernoulli matrix method has been used to find the approximate solutions of two-dimensional hyperbolic telegraph equations [18], linear partial differential equations [19], pantograph equation [20], nonlinear fuzzy Hammerstein-Volterra delay integral equations [21], fractional Fredholem-Volterra integro-differential equations [22], the Blasius and MHD Falkner-Skan boundary-layer equations [23], linear multidimensional diffusion and wave equations [24], optimal control problems [25] and Fuzzy integral equations [26]. Recently, El-Gamel and Adel [27] proposed a new approach to solving higher-order boundary value problems via Euler matrix method.

The rest of this article is organized as follows. In Section 2, we describe the wavelet-Galerkin method. In Section 3, Bernoullicollocation method is introduced. Section 4, gives specific three examples to test the two proposed methods and compare the results. Closing with conclusion In Section 5 . 


\section{Wavelet Bases}

\subsection{Governing Equation}

Setting

$$
\frac{\partial^{\alpha} u}{\partial t^{\alpha}} \approx \frac{u^{i+1}-\alpha u^{i}+(\alpha-1) u^{i-1}}{(\triangle t)^{\alpha}}
$$

Equation (2) may be approximated by

$$
\left[\frac{u^{i+1}-\alpha u^{i}+(\alpha-1) u^{i-1}}{(\Delta t)^{\alpha}}\right]=\frac{d^{2} u^{i+1}}{d x^{2}}+\eta\left(x, t^{i+1}\right)
$$

where $t^{i}=i \Delta t, i=0,1,2, \ldots$. Then, rewriting Equation (5)

$$
\frac{d^{2} u^{i+1}}{d x^{2}}-\left[\frac{1}{(\Delta t)^{\alpha}}\right] u^{i+1}=M\left(x, t^{i+1}\right)
$$

where

$$
M\left(x, t^{i+1}\right)=-\eta\left(x, t^{i+1}\right)-\left[\frac{\alpha}{(\Delta t)^{\alpha}}\right] u^{i}+\left[\frac{(\alpha-1)}{(\Delta t)^{\alpha}}\right] u^{i-1} .
$$

We assume that

$$
\eta(x, t)=h(t) z(x)=\sum_{i=0}^{m} c_{i} x^{i} h(t) .
$$

\subsection{Daubechies Wavelet Bases}

More detailed discussions about Daubechies wavelets can be found in $[28-31]$.

\subsection{Wavelet-Galerkin Method}

Let the solution $u_{J}(x)$ at the $(i+1)^{t h}$ time level of the problem be approximated by

$$
u_{J}(x)=2^{J / 2} \sum_{k=2-D}^{2^{J}-1} a_{k} \phi\left(2^{J} x-k\right), \quad \mathrm{k} \in Z
$$

By substituting the solution $u_{J}(x)$ in Equation (6), yields

$$
\begin{aligned}
& 2^{J / 2} \sum_{k=2-D}^{2^{J}-1} a_{k} \frac{d^{2}}{d x^{2}}\left[\phi\left(2^{J} x-k\right)\right]-2^{J / 2}\left[\frac{1}{(\Delta t)^{\alpha}}\right] \sum_{k=2-D}^{2^{J}-1} a_{k} \phi\left(2^{J} x-k\right) \\
& =M\left(x, t^{i+1}\right) .
\end{aligned}
$$

We use the inner product of both sides of Equation (8) with $2^{J / 2} \phi\left(2^{J} x-l\right)$ leads the following equation

$$
\sum_{k=2-D}^{2^{J}-1} b_{k l}^{J} a_{k}-\left[\frac{1}{(\Delta t)^{\alpha}}\right] \sum_{k=2-D}^{2^{J}-1} c_{k l}^{J} a_{k}=d_{m l}^{J}, l=2-D, 3-D, \ldots, 2^{J}-1 .
$$

where

$$
\begin{aligned}
b_{k l}^{J} & =2^{J} \int_{0}^{1} \phi\left(2^{J} x-k\right) \phi\left(2^{J} x-l\right) d x \\
& =\int_{-l}^{2^{J}-l} \phi(y-(k-l)) \phi(y) d y \\
& =\Gamma_{k-l}^{0}\left(2^{J}-l\right)-\Gamma_{k-l}^{0}(-l),
\end{aligned}
$$




$$
\begin{aligned}
c_{k l}^{J} & =2^{3 J} \int_{0}^{1} \phi^{\prime \prime}\left(2^{J} x-k\right) \phi\left(2^{J} x-l\right) d x \\
& =2^{2 J} \int_{-l}^{2^{J}-l} \phi^{\prime \prime}(y-(k-l)) \phi(y) d y \\
& =2^{2 J}\left[\Gamma_{k-l}^{2}\left(2^{J}-l\right)-\Gamma_{k-l}^{2}(-l)\right],
\end{aligned}
$$

and

$$
\begin{aligned}
d_{m l}^{J} & =\int_{0}^{1}\left[-\eta\left(x, t^{i+1}\right)-\left[\frac{\alpha}{(\Delta t)^{\alpha}}\right] u^{i}+\left[\frac{(\alpha-1)}{(\Delta t)^{\alpha}}\right] u^{i-1}\right] \phi_{J l}(x) d x \\
& =\int_{0}^{1}\left[-h\left(t^{i+1}\right) \sum_{i=0}^{m} c_{i} x^{i}-\left[\frac{\alpha}{(\Delta t)^{\alpha}}\right] u^{i}\right. \\
& +\left[\frac{(\alpha-!)}{(\Delta t)^{\alpha}}\right] u^{i-1} \phi_{J l}(x) d x \\
& =-h\left(t^{i+1}\right) \sum_{i=0}^{m} \int_{0}^{1} c_{i} x^{i} \phi_{J l}(x) d x-\int_{0}^{1}\left[\left[\frac{\alpha}{(\Delta t)^{\alpha}}\right] u^{i}\right. \\
& +\left[\frac{(\alpha-1)}{(\Delta t)^{\alpha}}\right] u^{i-1} \phi_{J l}(x) d x \\
& =-h\left(t^{i+1}\right) \sum_{i=0}^{m} \frac{c_{i}}{2^{\left(i+\frac{1}{2}\right) J}} M_{l}^{i}\left(2^{J}\right)-\frac{c_{00}}{2^{\left(\frac{1}{2}\right) J}} M_{l}^{0}\left(2^{J}\right) .
\end{aligned}
$$

The algorithm for calculating $\Gamma_{k-l}^{0}, \Gamma_{k-l}^{2}$, and $M_{l}^{m}$ has been described in [32].

The matrix-vector form of the Equation (9) is

$$
\left[\mathrm{W}-\left[\frac{1}{(\Delta t)^{\alpha}}\right] \mathrm{R}\right] \mathrm{A}=\mathrm{S}
$$

where

$$
\begin{aligned}
& \mathrm{W}=\left[b_{k l}^{J}\right]_{2-D \leq k, l \leq 2^{J}-1}, \quad \mathrm{R}=\left[c_{k l}^{J}\right]_{2-D \leq k, l \leq 2^{J}-1}, \\
& \mathrm{~S}=\left[d_{m l}^{J}\right]_{2-D} \leq l \leq 2^{J}-1, \quad \mathrm{~A}=\left[a_{2-D}, a_{3-D}, \ldots, a_{2^{J}-1}\right]^{t},
\end{aligned}
$$

where $t$ denotes the matrix transpose. Now we have a linear algebraic system that can be solved by the Q-R method.

\section{Bernoulli Bases}

More detailed discussions about Bernoulli operational matrix can be found in $[18,22,23,25]$.

\section{Bernoulli-Collocation Method}

Let the solution of (6) is

$$
u_{N}\left(x, t^{i+1}\right)=u_{N}(x)^{i+1} \simeq \sum_{n=0}^{N} a_{n} B_{n}(x)=\mathrm{B}(x) \mathrm{A}
$$

where

$$
\begin{aligned}
\mathrm{A}^{t} & =\left[a_{0}, a_{1}, \ldots, a_{N}\right] \\
\mathrm{B}(x) & =\left[B_{0}(x), B_{1}(x), \ldots, B_{N}(x)\right]
\end{aligned}
$$


then the matrix form of the second derivative is

$$
\frac{d^{2}}{d x^{2}} u_{N}\left(x, t^{i+1}\right)=\frac{d^{2}}{d x^{2}} u_{N}(x)^{i+1}=\mathrm{B}^{(2)}(x) \mathrm{A}=\mathrm{B}(x)\left(\mathrm{M}^{t}\right)^{2} \mathrm{~A} .
$$

where $\mathrm{M}$ is $(N+1) \times(N+1)$ Bernoulli operational matrices of differentiation described by

$$
\mathrm{M}=\left[\begin{array}{ccccc}
0 & 0 & \ldots & & 0 \\
1 & 0 & \ldots & & 0 \\
0 & 2 & \ldots & & 0 \\
\vdots & \vdots & \ddots & & \vdots \\
0 & \ldots & & N & 0
\end{array}\right] \text { and } \mathrm{B}(x)^{t}=\left[\begin{array}{c}
B_{0}(x) \\
B_{1}(x) \\
\vdots \\
B_{N-1}(x) \\
B_{N}(x)
\end{array}\right]
$$

By replacing each term in Equation (6) with the approximation defined in Equation (14) and (15) and collocate them at $x=x_{k}$ defined as the equal collocation points where

$$
x_{k}=\frac{k}{N}, \quad k=0,1,2, \ldots, N .
$$

We reach the following theorem

Theorem 1. If the assumed approximate solution of the boundaryvalue problem (6) is (14), then the discrete Bernoulli system is given by

$$
\begin{gathered}
{\left[\sum_{n=0}^{N} B_{n}^{\prime \prime}\left(x_{k}\right)-\frac{1}{(\Delta t)^{\alpha}} \sum_{n=0}^{N} B_{n}\left(x_{k}\right)\right] a_{n}} \\
=-\eta\left(x_{i}, t^{i+1}\right)-\frac{\alpha}{(\Delta t)^{\alpha}} u\left(x_{k}, t^{i}\right)+\frac{(\alpha-1)}{(\Delta t)^{\alpha}} u\left(x_{k}, t^{i-1}\right) .
\end{gathered}
$$

Proof. By replacing each term of Equation (6) with the corresponding approximation represented in Equation (14) and (15) and collocate them with $x=x_{k}$ collocation points.

The fundamental matrix for the above system is

$$
\Phi \mathrm{A}=\mathrm{F}
$$

where

$$
\Phi=\mathrm{B}\left(\mathrm{M}^{t}\right)^{2}-\mathrm{JB},
$$

and

$$
\begin{gathered}
\mathrm{B}=\left(\begin{array}{ccccc}
B_{0}\left(x_{0}\right) & B_{1}\left(x_{0}\right) & B_{2}\left(x_{0}\right) & \ldots & B_{N}\left(x_{0}\right) \\
B_{0}\left(x_{1}\right) & B_{1}\left(x_{1}\right) & B_{2}\left(x_{1}\right) & \ldots & B_{N}\left(x_{1}\right) \\
B_{0}\left(x_{2}\right) & B_{1}\left(x_{2}\right) & B_{2}\left(x_{2}\right) & \ldots & B_{N}\left(x_{2}\right) \\
\vdots & \vdots & \vdots & \ddots & \vdots \\
B_{0}\left(x_{N}\right) & B_{1}\left(x_{N}\right) & B_{2}\left(x_{N}\right) & \ldots & B_{N}\left(x_{N}\right)
\end{array}\right) \\
\mathrm{F}=\left(\begin{array}{c}
-\eta\left(x_{0}, t^{i+1}\right)-\frac{\alpha}{(\Delta t)^{\alpha}} u\left(x_{0}, t^{i}\right)+\frac{(\alpha-1)}{(\Delta t)^{\alpha}} u\left(x_{0}, t^{i-1}\right) \\
-\eta\left(x_{1}, t^{i+1}\right)-\frac{\alpha}{(\Delta t)^{\alpha}} u\left(x_{1}, t^{i}\right)+\frac{(\alpha-1)}{(\Delta t)^{\alpha}} u\left(x_{1}, t^{i-1}\right) \\
\vdots \\
-\eta\left(x_{N}, t^{i+1}\right)-\frac{\alpha}{(\Delta t)^{\alpha}} u\left(x_{N}, t^{i}\right)+\frac{(\alpha-1)}{(\Delta t)^{\alpha}} u\left(x_{N}, t^{i-1}\right)
\end{array}\right)
\end{gathered}
$$




$$
\mathrm{J}=\left(\begin{array}{ccccc}
\frac{1}{(\Delta t)^{\alpha}} & 0 & 0 & \cdots & 0 \\
0 & \frac{1}{(\Delta t)^{\alpha}} & 0 & \ldots & 0 \\
0 & 0 & \frac{1}{(\Delta t)^{\alpha}} & \cdots & 0 \\
\vdots & \vdots & \vdots & \ddots & \vdots \\
0 & 0 & 0 & \cdots & \frac{1}{(\Delta t)^{\alpha}}
\end{array}\right) .
$$

Then, substituting the approximation from (14) in the boundary conditions in Equation (3) yields

$$
\sum_{n=0}^{N} a_{n} B_{n}(0)=h_{1}\left(t^{i+1}\right)
$$

and

$$
\sum_{n=0}^{N} a_{n} B_{n}(1)=h_{2}\left(t^{i+1}\right) .
$$

Replacing the first and the last row in the augmented matrix $[\Phi ; \mathrm{F}]$ with the boundary conditions from Equations (17) and (18) will lead to the new augmented matrix $[\Theta ; \tilde{F}]$ which is an $N+1$ linear equations in $N+1$ unknowns defined as

$$
\Theta \mathrm{A}=\tilde{F}
$$

where

$$
\begin{gathered}
\Theta=\left(\begin{array}{ccccc}
B_{0}(0) & B_{1}(0) & B_{2}(0) & \ldots & B_{N}(0) \\
& & \vdots & & \\
\ldots & \ldots & \Phi & \ldots & \ldots \\
& & \vdots & & \\
B_{0}(1) & B_{1}(1) & B_{2}(1) & \ldots & B_{N}(1)
\end{array}\right), \\
\tilde{F}=\left(\begin{array}{c}
-h_{1}\left(t^{i+1}\right) \\
-\eta\left(x_{0}, t^{i+1}\right)-\frac{\alpha}{(\Delta t)^{\alpha}} u\left(x_{0}, t^{i}\right)+\frac{(\alpha-1)}{(\Delta t)^{\alpha}} u\left(x_{0}, t^{i-1}\right) \\
-\eta\left(x_{1}, t^{i+1}\right)-1 \frac{\alpha}{(\Delta t)^{\alpha}} u\left(x_{1}, t^{i}\right)+\frac{(\alpha-1)}{(\Delta t)^{\alpha}} u\left(x_{1}, t^{i-1}\right) \\
\vdots \\
-\eta\left(x_{N}, t^{i+1}\right)-\frac{\alpha}{(\Delta t)^{\alpha}} u\left(x_{N}, t^{i}\right)+\frac{(\alpha-1)}{(\Delta t)^{\alpha}} u\left(x_{N}, t^{i-1}\right) \\
h_{2}\left(t^{i+1}\right) .
\end{array}\right)
\end{gathered}
$$

This system is solved using the Q-R method for finding the unknown coefficients $\left\{a_{n}\right\}_{n=0}^{N}$, but with known approximate $u\left(x_{k}, t^{i-1}\right)$ and then $u\left(x_{k}, t^{i}\right)$ can be evaluated, respectively.

\section{Numerical Examples}

Three examples are considered to demonstrate the efficiency and accuracy of the proposed methods in homogeneous and nonhomogeneous boundary conditions. Daubechies 6 wavelet, $D=6$, is used and each example was run for $J=9$ and we take $\triangle t=0.001$. The maximum absolute error is taken as

$$
\left\|\mathrm{E}_{W G}\right\|=\left|u_{\text {exact }}-u_{\text {wavelet-Galerkin }}\right|,
$$

and

$$
\left\|\mathrm{E}_{B C}\right\|=\left|u_{\text {exact }}-u_{\text {Bernoulli-collocation }}\right| .
$$

Example 1 [17] Consider the following problem

$$
u_{t}=u_{x x} \quad 0 \leq x \leq 1, t>0,
$$


subject to the boundary conditions

$$
u(0, t)=\exp (t), \quad u(1, t)=\exp (t+1),
$$

and the initial condition

$$
u(x, 0)=\exp (x),
$$

whose exact solution is

$$
u(x, t)=\exp (t+x) .
$$

Table 1 shows the comparison between the absolute errors $\left\|\mathrm{E}_{B C}\right\|$ and $\left\|\mathrm{E}_{W G}\right\|$.

Table 1. Maximum absolute errors for Example 1.

\begin{tabular}{ccc}
\hline$(x, t)$ & $\left\|\mathbf{E}_{B C}\right\|, N=10$ & $\left\|\mathbf{E}_{W G}\right\|, J=9$. \\
\hline$(0.25,0.1)$ & $4.631913 \mathrm{E}-06$ & $8.5436 \mathrm{E}-05$ \\
$(0.5,0.1)$ & $6.838514 \mathrm{E}-06$ & $9.3299 \mathrm{E}-05$ \\
$(0.75,0.1)$ & $6.751201 \mathrm{E}-06$ & $1.7983 \mathrm{E}-05$ \\
$(0.25,0.5)$ & $5.340952 \mathrm{E}-05$ & $5.4634 \mathrm{E}-04$ \\
$(0.5,0.5)$ & $7.813925 \mathrm{E}-05$ & $7.6003 \mathrm{E}-04$ \\
$(0.75,0.5)$ & $6.350286 \mathrm{E}-05$ & $5.7020 \mathrm{E}-04$ \\
$(0.25,1.0)$ & $1.769145 \mathrm{E}-05$ & $1.3965 \mathrm{E}-03$ \\
$(0.5,1.0)$ & $2.588038 \mathrm{E}-05$ & $1.7219 \mathrm{E}-03$ \\
\hline
\end{tabular}

Example 2 [17] Consider the following problem

$$
u_{t}=u_{x x} \quad 0 \leq x \leq 1, t>0,
$$

subject to the boundary conditions

$$
u(0, t)=0, \quad u(1, t)=\sin 1 e^{-t},
$$

and the initial conditions

$$
u(x, 0)=\sin x
$$

whose exact solution is

$$
u(x, t)=\sin x e^{-t} .
$$

The computational results are summarized in Table 2 .

Table 2. Comparison between the maximum absolute errors for Example 2 .

\begin{tabular}{ccc}
\hline$(x, t)$ & $\left\|\mathbf{E}_{B C}\right\|, N=10$ & $\left\|\mathbf{E}_{W G}\right\| J=9$. \\
\hline$(0.25,0.1)$ & $1.00112 \mathrm{E}-06$ & $3.2750 \mathrm{E}-05$ \\
$(0.5,0.1)$ & $1.70939 \mathrm{E}-06$ & $5.5452 \mathrm{E}-05$ \\
$(0.75,0.1)$ & $1.58965 \mathrm{E}-06$ & $4.3456 \mathrm{E}-05$ \\
$(0.25,0.5)$ & $6.18197 \mathrm{E}-06$ & $1.9567 \mathrm{E}-05$ \\
$(0.5,0.5)$ & $9.80818 \mathrm{E}-06$ & $5.4572 \mathrm{E}-04$ \\
$(0.75,0.5)$ & $8.19902 \mathrm{E}-06$ & $7.7654 \mathrm{E}-04$ \\
$(0.25,1.0)$ & $7.60298 \mathrm{E}-06$ & $1.6562 \mathrm{E}-04$ \\
$(0.5,1.0)$ & $1.20468 \mathrm{E}-05$ & $3.7982 \mathrm{E}-03$ \\
$(0.75,1.0)$ & $1.00501 \mathrm{E}-05$ & $4.4324 \mathrm{E}-03$ \\
\hline
\end{tabular}


Example 3 [17] Consider the following problem

$$
u_{t t}=u_{x x}+2 \exp (-\pi t) \sin (\pi x), \quad 0 \leq x \leq 1, t>0,
$$

subject to the boundary conditions

$$
u(0, t)=0, \quad u(1, t)=0,
$$

and the initial conditions

$$
\begin{aligned}
u(x, 0) & =\sin (\pi x) \\
u_{t}(x, 0) & =-\pi \sin (\pi x)
\end{aligned}
$$

whose exact solution is

$$
u(x, t)=\exp (-\pi t) \sin (\pi x) .
$$

The computational results are summarized in Table 3.

Table 3. Comparison between the maximum absolute errors for Example 3.

\begin{tabular}{ccc}
\hline$(x, t)$ & $\left\|\mathbf{E}_{B C}\right\|, N=10$ & $\left\|\mathbf{E}_{W G}\right\| J=9$. \\
\hline$(0.25,0.1)$ & $4.64123 \mathrm{E}-05$ & $4.7799 \mathrm{E}-03$ \\
$(0.5,0.1)$ & $6.23950 \mathrm{E}-05$ & $3.8654 \mathrm{E}-03$ \\
$(0.75,0.1)$ & $4.64123 \mathrm{E}-05$ & $2.7143 \mathrm{E}-03$ \\
$(0.25,0.5)$ & $1.28684 \mathrm{E}-04$ & $6.5798 \mathrm{E}-02$ \\
$(0.5,0.5)$ & $1.72999 \mathrm{E}-04$ & $7.3478 \mathrm{E}-02$ \\
$(0.75,0.5)$ & $1.28684 \mathrm{E}-04$ & $1.8764 \mathrm{E}-02$ \\
$(0.25,1.0)$ & $1.13949 \mathrm{E}-03$ & $3.2385 \mathrm{E}-01$ \\
$(0.5,1.0)$ & $1.63140 \mathrm{E}-03$ & $5.7492 \mathrm{E}-01$ \\
$(0.75,1.0)$ & $1.13949 \mathrm{E}-03$ & $4.2954 \mathrm{E}-01$ \\
\hline
\end{tabular}

\section{Conclusion}

The main objective of this article is to develop two accurate methods to solve nonhomogeneous heat and wave equations. Bernoulli operational matrix with collocation method and wavelet with Galerkin method have reduced the problem into the linear algebraic system. Some illustrative problems are given to ensure the high efficiency of the proposed algorithms.

\section{Acknowledgements}

The authors would like to thank the referees for the valuable suggestions and comments.

\section{Conflicts of Interest}

The authors declare no conflicts of interest regarding the publication of this paper.

\section{References}

[1] Wang, Y., Chenb, X. and He, Z. (2012) A Second-Generation Wavelet-Based Finite Element Method for the Solution of Partial Differential Equations. Applied Mathematics Letters, 25, 16081613.

https://doi.org/10.1016/j.aml.2012.01.021 
[2] Xu, J. and Shann, W. (1992) Galerkin-Wavelet Methods for TwoPoint Boundary Value Problems. Numerische Mathematik, 63, 123-144.

https://doi.org/10.1007/BF01385851

[3] Avudainayagam, A. (2000) Wavelet-Galerkin Method for IntegroDifferential Equations. Applied Numerical Mathematics, 32, 247254.

https://doi.org/10.1016/S0168-9274(99)00026-4

[4] Beylkin, G., Coifman, R. and Rokhlin, V. (1991) Fast Wavelet Transform and Numerical Algorithm I. Communications on Pure and Applied Mathematics, 44, 141-183.

https://doi.org/10.1002/cpa.3160440202

[5] Fang, W., Wang, Y. and Xu, Y. (2004) An Implementation of Fast Wavelet-Galerkin Methods for Integral Equations of the Second Kind. Journal of Scientific Computing, 20, 277-302. https://doi.org/10.1023/B:JOMP.0000008723.85496.ce

[6] Alpert, B. (1993) A Class of Bases in $l_{2}$ for the Sparse Representation of Integral Operators. SIAM Journal on Mathematical Analysis, 24, 246-262.

https://doi.org/10.1137/0524016

[7] El-Gamel, M. (2004) Wavelet Algorithm for the Numerical Solution of Nonhomogeneous Time-Dependent Problems. Journal of Difference Equations and Applications, 9, 169-185.

[8] El-Gamel, M. (2006) A Wavelet-Galerkin Method for a Singularly Perturbed Convection-Dominated Diffusion Equation. Applied Mathematics and Computation, 181, 1635-1644.

https://doi.org/10.1016/j.amc.2006.03.017

[9] El-Azab, M. and El-Gamel, M. (2007) A Numerical Algorithm for the Solution of Telegraph Equations. Applied Mathematics and Computation, 190, 757-764.

https://doi.org/10.1016/j.amc.2007.01.091

[10] Panigrahi, B. and Nelakanti, G. (2012) Wavelet-Galerkin Method for Eigenvalue Problem of a Compact Integral Operator. Applied Mathematics and Computation, 218, 1222-1232.

https://doi.org/10.1016/j.amc.2011.05.114

[11] Rathish, B. and Priyadarshi, G. (2018) Wavelet Galerkin Method for Fourth-Order Multi-Dimensional Elliptic Partial Differential Equations. International Journal of Wavelets, Multiresolution and Information Processing, 16, 185-205.

https://doi.org/10.1142/S0219691318500455

[12] Priyadarshi, G. and Rathish, B. (2018) Wavelet-Galerkin Method for Fourth Order Linear and Nonlinear Differential Equations. Applied Mathematics and Computation, 327, 8-21. https://doi.org/10.1016/j.amc.2017.12.047

[13] Mohmmadi, F. (2016) Wavelet-Galerkin Method for Solving Stochastic Fractional Differential Equations. Journal of Fractional Calculus and Applications, 7, 73-86. 
[14] Wang, J., Liu, X. and Zhou, Y. (2018) A High-Order Accurate Wavelet Method for Solving Schrdinger Equations with General Nonlinearity. Applied Mathematics and Mechanics, 39, 275-290. https://doi.org/10.1007/s10483-018-2299-6

[15] Yang, Z. and Liao, S. (2018) On the Generalized WaveletGalerkin Method. Journal of Computational and Applied Mathematics, 331, 178-195.

https://doi.org/10.1016/j.cam.2017.09.042

[16] El-Gamel, M. and Zayed, A. (2002) A Comparison between the Wavelet-Galerkin and the Sinc-Galerkin Methods in Solving Nonhomogeneous Heat Equations. In: Nashed, Z. and Scherzer, O., Eds., Contemporary Mathematics of the American Mathematical Society, Series, Inverse Problem, Image Analysis, and Medical Imaging, Vol. 313, AMS, Providence.

[17] El-Gamel, M. (2007) Comparison of the Solutions Obtained by Adomian Decomposition and Wavelet-Galerkin Methods of Boundary-Value Problems. Applied Mathematics and Computation, 186, 652-664.

https://doi.org/10.1016/j.amc.2006.08.010

[18] Singh, S., Kumar Patel, V., Kumar Singh, V. and Tohidi, E. (2018) Application of Bernoulli Matrix Method for Solving Two-Dimensional Hyperbolic Telegraph Equations with Dirichlet Boundary Conditions. Computers \& Mathematics with Applications, 75, 2280-2294.

https://doi.org/10.1016/j.camwa.2017.12.003

[19] Toutounian, F. and Tohidi, E. (2013) A New Bernoulli Matrix Method for Solving Second Order Linear Partial Differential Equations with the Convergence Analysis. Applied Mathematics and Computation, 223, 298-310.

https://doi.org/10.1016/j.amc.2013.07.094

[20] Tohidi, E., Bhrawy, A. and Erfani, K. (2007) A Collocation Method Based on Bernoulli Operational Matrix for Numerical Solution of Generalized Pantograph Equation. Applied Mathematical Modelling, 37, 4283-4294.

https://doi.org/10.1016/j.apm.2012.09.032

[21] Sahu, P. and SahaRay, S. (2017) A New Bernoulli Wavelet Method for Accurate Solutions of Nonlinear Fuzzy HammersteinVolterra Delay Integral Equations. Fuzzy Sets and Systems, 309, 131-144.

https://doi.org/10.1016/j.fss.2016.04.004

[22] Rahimkhan, P., Ordokhani, Y. and Babolian, E. (2017) Fractional-Order Bernoulli Functions and Their Applications in Solving Fractional Fredholem-Volterra Integro-Differential Equations. Applied Numerical Mathematics, 122, 66-81.

https://doi.org/10.1016/j.apnum.2017.08.002

[23] Calvert, V. and Razzaghi, M. (2017) Solutions of the Blasius and MHD Falkner-Skan Boundary-Layer Equations by Modified Rational Bernoulli Functions. International Journal of Numerical Methods for Heat \& Fluid Flow, 27, 1687-1705.

https://doi.org/10.1108/HFF-05-2016-0190 
[24] Zoghe, B., Tohidi, E. and Shatey, S. (2017) Bernoulli-Collocation Method for Solving Linear Multidimensional Diffusion and Wave Equations with Dirichlet Boundary Conditions. Advances in Mathematical Physics, 2017, Article ID: 5691452.

https://doi.org/10.1155/2017/5691452

[25] Bokhari, A., Amir, A., Bahri, S. and Belgacem, F. (2017) A Generalized Bernoulli Wavelet Operational Matrix of Derivative Applications to Optimal Control Problems. Nonlinear Studies, 24, 775-790.

[26] Mohamed, A. and Mohamed, R. (2018) Numerical Solution of Fuzzy Integral Equations via a New Bernoulli-Wavelet Method. International Journal of Modern Mathematical Sciences, 16, 3750.

[27] El-Gamel, M. and Adel, W. (2018) Numerical Investigation of the Solution of Higher-Order Boundary Value Problems via Euler Matrix Method. SeMA Journal, 75, 349-364.

https://doi.org/10.1007/s40324-017-0136-y

[28] Amaratunga, K., Williams, J., Qian, S. and Weiss, J. (1994) Wavelet-Galerkin Solutions for One-Dimensional Partial Differential Equations. International Journal for Numerical Methods in Engineering, 37, 2703-2716.

https://doi.org/10.1002/nme.1620371602

[29] Mallat, S. (1989) Multiresoltion Approximations and Wavelet Orthonormal Bases of $L^{2}(R)$. Transactions of the American Mathematical Society, 315, 69-87.

[30] Daubechies, I. (1992) Ten Lectures on Wavelets. Captial City Press, Vermont.

[31] Qian, S. and Weiss, J. (1993) Wavelets and the Numerical Solution of Boundary Value Problems. Applied Mathematics Letters, 6, $47-52$.

https://doi.org/10.1016/0893-9659(93)90147-F

[32] Chen, M., Wang, C. and Shin, Y. (1996) The Computation of Wavelet-Galerkin Approximation on a Bounded Interval. International Journal for Numerical Methods in Engineering, 39, 2921-2944.

https://doi.org/10.1002/(SICI)1097-0207(19960915)39:17〈2921:: AID-NME983 $>3.0 . C O ; 2-\mathrm{D}$ 